\title{
Enhanced photo bio-reaction by multiscale bubbles
}

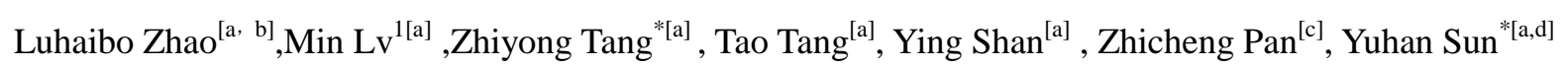

${ }^{a}$ Key Laboratory of Low-Carbon Conversion Science and Engineering, Shanghai Advanced Research Institute (SARI), Chinese Academy of Sciences (CAS), Shanghai 201210, China

${ }^{\mathrm{b}}$ University of Chinese Academy of Sciences, Beijing, 100049, China

${ }^{\mathrm{c}}$ Key Laboratory of Energy Thermal Conversion and Control of Ministry of Education, School of Energy and Environment, Southeast University, Nanjing 210096,China

${ }^{\mathrm{d}}$ School of Physical Science and Technology, Shanghai Tech University, Shanghai 201210, China

${ }^{1}$ Contributed equally to this work ;Z.Y. Tang and Y.H. Sun are the corresponding authors for this article.Y.H. Sun Email: sunyh@ sari.ac.cn.

\section{Abstract}

Based on experimental and computational fluid dynamics simulations, the scale effect between

the bubbles and the algae for different sizes in the culture process were investigated in a

photobioreactor column under single and combined gas intake conditions, including factors such as the bubble distribution, gas holdup and mass transfer coefficient of different scale bubbles (500 $\mu \mathrm{m}$ microbubbles and $5 \mathrm{~mm}$ bubbles). In general, microbubbles could improve mass transfer and photo bio-reaction. For microalgae cultures with large particle sizes or high biomass concentration, the mixing ability of microbubbles was weakened, and therefore, the culture efficiency decreased. However, this can be improved by using millimeter bubbles with the strong turbulent characteristic. Therefore, a multiscale bubble combination intake method was proposed. This method can significantly strengthen both the multiphase mixture and mass transfer 
processes and was beneficial to improving the growth efficiency of Chlorella.

Keywords: photobioreactor, multiscale, microbubble, mass transfer, CFD

\section{Nomenclature}

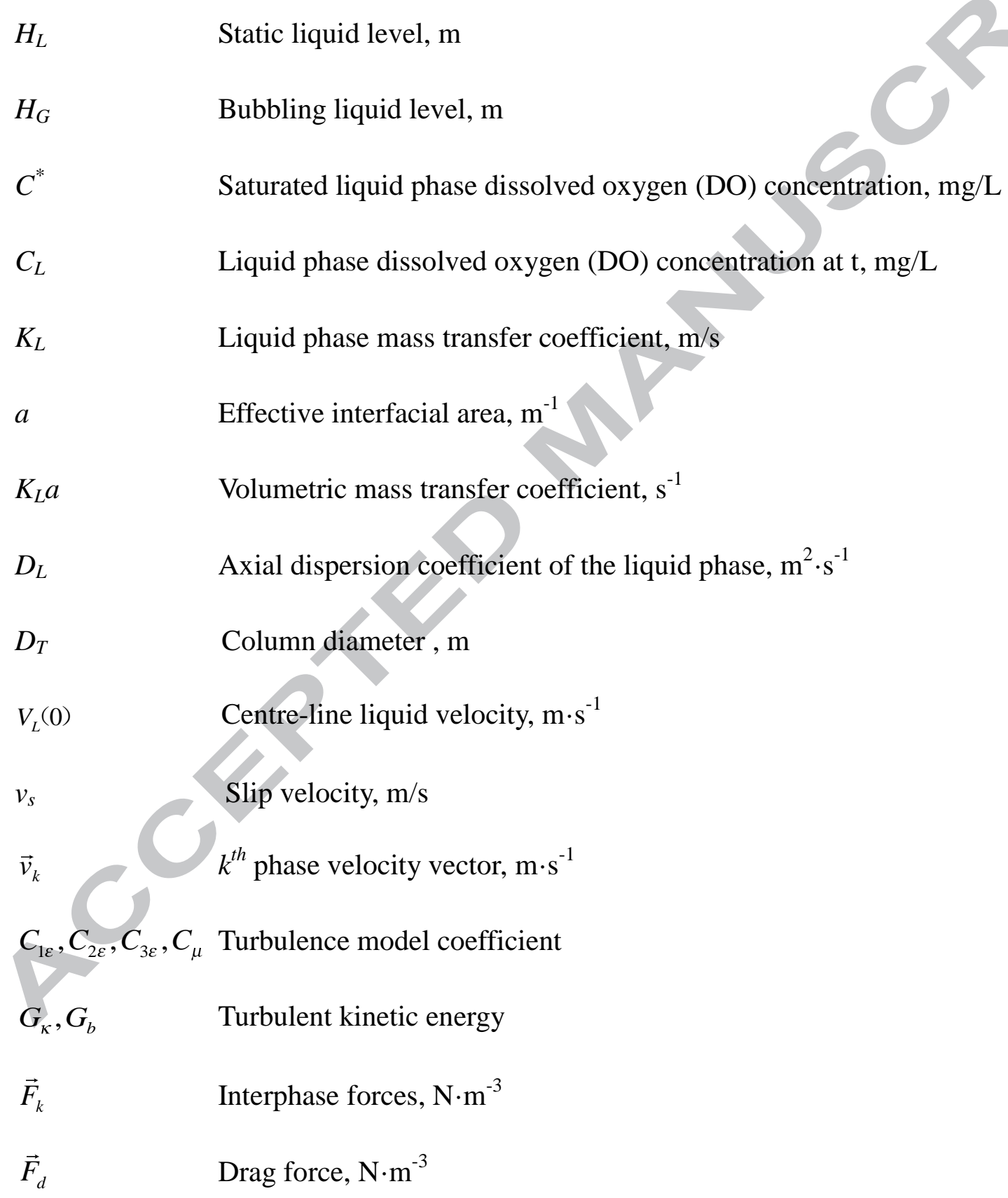




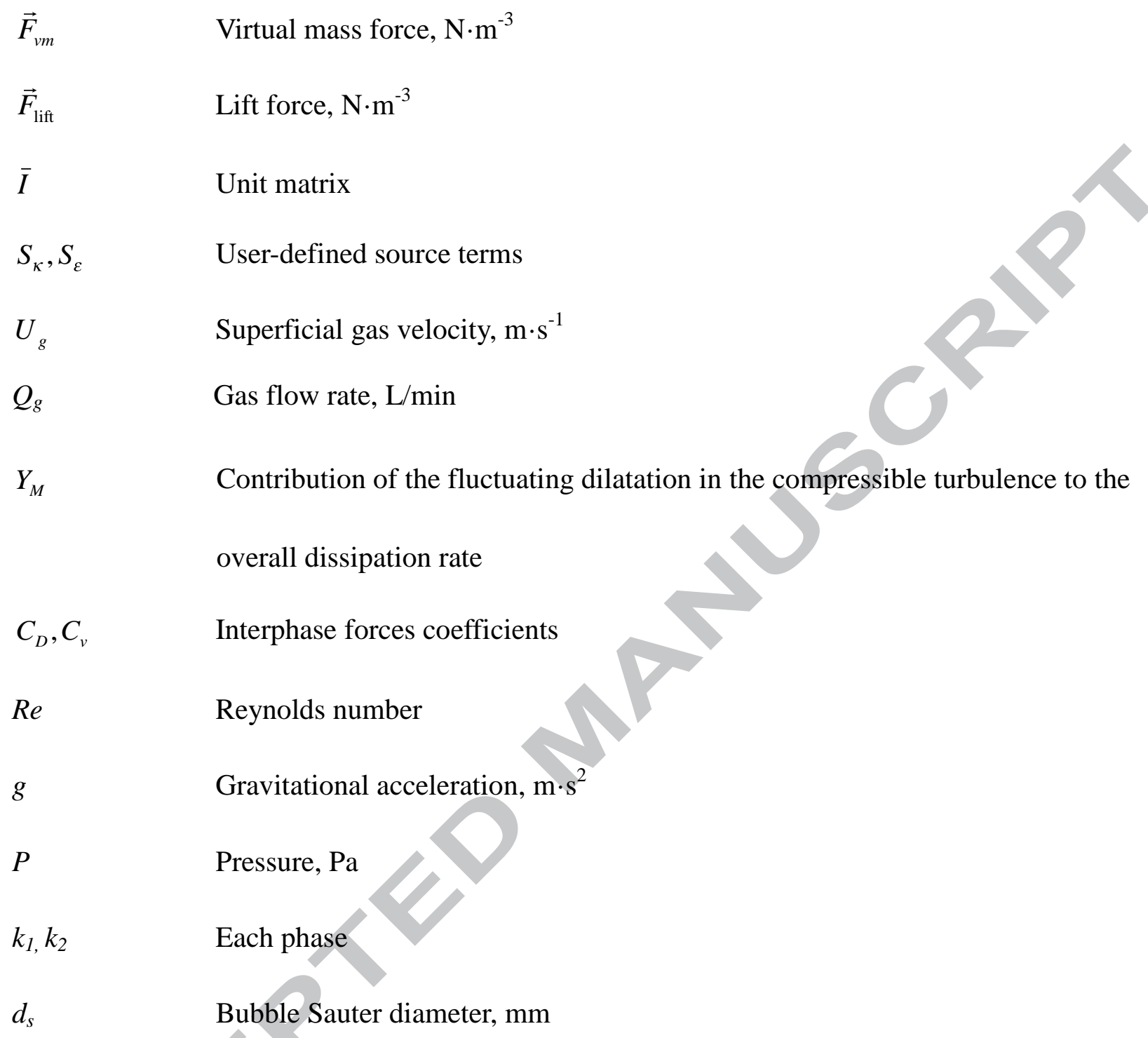

Greek letters

$\alpha_{k} \quad k^{\text {th }}$ phase volume fraction

$v_{L} \quad$ Kinematic viscosity of the liquid phase, $\mathrm{m}^{2} \cdot \mathrm{s}^{-1}$

$\varepsilon \quad$ Turbulent dissipation rate, $\mathrm{m}^{2} \cdot \mathrm{s}^{-3}$

$\kappa \quad$ Turbulent kinetic energy, $\mathrm{m}^{2} \cdot \mathrm{s}^{-2}$

$\rho_{k} \quad k^{t h}$ phase density, $\mathrm{kg} \cdot \mathrm{m}^{3}$

$\bar{\tau}_{k} \quad$ Stress-strain tensor, $\mathrm{N} \cdot \mathrm{m}^{-2}$ 


$\begin{array}{ll}\mu_{k} & \text { Shear viscosity, } \mathrm{Pa} \cdot \mathrm{s} \\ \lambda_{k} & \text { Bulk viscosity, } \mathrm{Pa} \cdot \mathrm{s} \\ \mu_{t} & \text { Turbulent viscosity, } \mathrm{Pa} \cdot \mathrm{s} \\ \sigma_{\kappa}, \sigma_{\varepsilon} & \text { Turbulent Prandtl numbers }\end{array}$

Abbreviations

PBR

Photobioreactor

CFD

Computational fluid dynamics

dw dry weight 


\section{Introduction}

Microalgae have advantages of high photosynthetic efficiency, fast growth rates, and the possibility of large-scale cultures, which can be coupled with other engineering techniques (such as wastewater treatment and biological carbon sequestration). Photobioreactors (PBRs) are special devices that can be used for photosynthesis in microorganisms, such as microalgae. They have systems to regulate and control the culture conditions, such as light, $\mathrm{CO}_{2}$, temperature, $\mathrm{pH}$ and nutrients. Open and closed types of microalgae PBRs exist[1-3]. Efficient design, optimization, and amplification of PBRs are bottlenecks in scaling-up of the microalgae culture[4]. The key issues are inhomogeneity of the gas-liquid-algae multiphase flow and low efficiency of the mass transfer and bio-reactions. Most of the existing methods for improving the PBR performance targeted the interaction mechanism between the three phases of gas-liquid-algae and the light transfer process[3]. Recently,Cao et al.[5-8] succeeded in studying a series of novel mathematical models to simulate temporal evolution of cells, light intensity and nutrients concentration within the growth medium as well as carbon dioxide and oxygen concentration in the liquid and gas phase, which improved microalgal cultures and contributed to the industrial scale in terms of studying the microalgal cells(like size distribution, disruption and multiple fission ). As well,it was also vital to understand the flow control mechanism of the bubbles in the algae solution, which mainly depended on gas-liquid mixing, bubble size, flow pattern, gas holdup, residence time, and gas-liquid mass transfer[9].

Many studies [3,9-11] have indicated that the mass transfer coefficient had an important impact on the performance of PBRs and other bubbling reactors. The mass transfer at gas-liquid 
reaction interfaces was influenced by the bubble size distribution, superficial gas velocity, and gas holdup. Zhang et al. stated that the bubble size was an important parameter that affected the interfacial mass transfer and the reaction rate[12]. When the bubble diameter was less than $0.8 \mathrm{~mm}$, the liquid mass transfer coefficient $K_{L}$ decreased rapidly, in contrast, when the bubble size decreased from 2 to $0.1 \mathrm{~mm}$, the gas-liquid interfacial area $a$ increased exponentially. The increase of $a$ was much higher than the decrease in $K_{L}$. As a result, the gas-liquid interfacial area dominated the total mass transfer rate. As the bubbles became smaller, the changes in $K_{L}$ and $a$ were opposite. Therefore, it was of great importance to strengthen the process of gas-liquid-algae mixing and reaction by studying the overall trend of $K_{L} a$ for different combination of bubble sizes. In recent years, microbubbles have been widely applied in environmental wastewater treatment as an important means of process intensification because of their advantages such as large contact area, high mass transfer efficiency and long residence time[13]. For example, Zimmerman et al. experimented with microbubbles (approximately $550 \mu \mathrm{m}$ in diameter)[14-15]. Their results demonstrated that the mass transfer efficiency of the $500 \mu \mathrm{m}$ bubbles increased by $29 \%$ compared with the 1-2 mm bubbles, and good results were obtained when cultivating Dunaliella (which has a cell size of $30 \mu \mathrm{m}$ ). However, due to the broad size distribution of microalgae (Chlorella is approximately $5 \mu \mathrm{m}$ in size and cyanobacteria has cell sizes of 100-300 $\mu \mathrm{m}$ ), the size effects between microbubbles and microalgae would have important influences on the flow mixing and mass transfer, which has seldom been reported. Most of studies on the bubbling PBRs were conducted with single millimeter-scale bubbles. Khoo et al. worked on the effects of hydrodynamics and gas-liquid mass transfer limitations on the biomass production of C. vulgaris 
at different compressed-air aeration rates with the traditional millimeter bubbles[16]. Manjrekar et al. illustrated the application of a 4-point optical probe technique to study millimeter-bubble dynamics in a photobioreactor[17]. Currently, few studies had focused on the multiphase flow of multiscale bubbles and its effects on the performance of microalgae cultivation. In this work, the flow behavior of different-sized bubbles in the PBRs and the size effect of bubbles and microalgae were studied. The effects of multiscale bubble flow, fluids mixing and mass transfer on the growth kinetics of microalgae were investigated.

\section{Materials and methods}

\subsection{Experimental apparatus and conditions}

The bubble-column PBR employed in this study was composed of Plexiglas. It had an inner diameter of $235 \mathrm{~mm}$ and a height of $1 \mathrm{~m}$. The wall thickness of the Plexiglas tube was $2 \mathrm{~mm}$, which was relatively thin to provide a clear view of the bubble behavior inside the reactor using a

high-speed camera. A gas distributor was installed at the bottom of the reactor,which consisted of a 5 - $\mu \mathrm{m}$ metal porous membrane distributor $(\mathrm{S} 1)$ and a porous plate $(\mathrm{S} 2)$ with an average pore size of $100 \mu \mathrm{m}$. In the experiments, the volume of the liquid phase was $18 \mathrm{~L}$, and the height of the liquid was approximately $400 \mathrm{~mm}$. Air was fed by the air compressor and the gas flow rate was controlled at the range of $0-10 \mathrm{~L} / \mathrm{min}$ using float flowmeters. The lighting was provided by a white light-emitting diode (LED) lamp with a light intensity of $120 \mu \mathrm{mol} / \mathrm{m}^{2} \cdot \mathrm{s}$. The hydrodynamic characteristics were measured in a two-phase system of air and water. A three-phase bubbling culture system was established using Chlorella and cyanobacteria with the 
modified BG11 as the medium[18], which was listed in the Table.1. The temperature was controlled at $21 \pm 1^{\circ} \mathrm{C}$. The dry weight of the biomass was obtained by filtration and baking at $100^{\circ} \mathrm{C}$ for 24 hours.

Table.1 Composition of the BG11 medium

\begin{tabular}{cccc}
\hline Composition & $\begin{array}{c}\text { Concentration } \\
(\mathrm{mg} / \mathrm{L})\end{array}$ & Composition & $\begin{array}{c}\text { Concentration } \\
(\mathrm{mg} / \mathrm{L})\end{array}$ \\
\hline $\mathrm{NaNO}_{3}$ & 1500 & $\left(\mathrm{NH}_{4}\right)_{3} \mathrm{FeC}_{12} \mathrm{H}_{10} \mathrm{O}_{14}$ & 6 \\
$\mathrm{~K}_{2} \mathrm{HPO}_{4} \cdot 3 \mathrm{H}_{2} \mathrm{O}$ & 52 & $\mathrm{C}_{10} \mathrm{H}_{14} \mathrm{~N}_{2} \mathrm{Na}_{2} \mathrm{O}_{8} \cdot 2 \mathrm{H}_{2} \mathrm{O}$ & 1 \\
$\mathrm{~K}_{2} \mathrm{HPO}_{4}$ & 40 & $\mathrm{H}_{3} \mathrm{BO}_{3}$ & 2.86 \\
$\mathrm{MgSO}_{4} \cdot 7 \mathrm{H}_{2} \mathrm{O}$ & 75 & $\mathrm{MnCl}_{2} \cdot 4 \mathrm{H}_{2} \mathrm{O}$ & 1.86 \\
$\mathrm{CaCl}_{2} \cdot 2 \mathrm{H}_{2} \mathrm{O}$ & 36 & $\mathrm{ZnSO}_{4} \cdot 7 \mathrm{H}_{2} \mathrm{O}$ & 0.22 \\
$\mathrm{CaCl}_{2}$ & 27 & $\mathrm{Na}_{2} \mathrm{MoO}_{4} \cdot 2 \mathrm{H}_{2} \mathrm{O}$ & 0.39 \\
$\mathrm{Na}_{2} \mathrm{CO}_{3}$ & 20 & $\mathrm{CuSO}_{4} \cdot 5 \mathrm{H}_{2} \mathrm{O}$ & 0.08 \\
$\mathrm{C}_{6} \mathrm{H}_{8} \mathrm{O}_{7} \cdot \mathrm{H}_{2} \mathrm{O}$ & 6 & $\mathrm{Co}_{\left(\mathrm{NO}_{3}\right)_{2} \cdot 6 \mathrm{H}_{2} \mathrm{O}}$ & 0.05 \\
\hline
\end{tabular}

\subsection{Bubbling flow characteristics in PBRs}

\subsubsection{Bubble size}

The bubble size distribution was measured using a non-intrusive high-speed camera. A series of images were taken and processed using a MATLAB imaging programme. The process included four steps: image enhancement, image segmentation, image morphological processing, and particle measurement[19]. The periodic bubble size distribution was obtained and averaged, and the Sauter radius was calculated using the following formula[20]: 
$d_{s}=\sum_{i=1}^{N} d_{i}^{3} / \sum_{i=1}^{N} d_{i}^{2}$

Mass transfer modeling and computational fluid dynamics (CFD) simulations were performed based on the above results.

\subsubsection{Residence time}

The residence time of the gas phase in the PBR was measured using the tracer method[21], a tracer (sodium hypochlorite solution) was introduced at the bubbling site. The $\mathrm{pH}$ measurement was taken at the liquid surface, and a graph of the $\mathrm{pH}$ values versus time was plotted after the injecting of the tracer.

\subsubsection{Gas holdup}

The gas holdup is the volume fraction of the bubbles, and it is an important parameter for characterizing the fluid dynamics in the multiphase bubbling reactor,which reflects the gas-liquid interfacial area and affects the mass transfer and reaction in the multiphase reactor. The gas holdup was measured using the volume expansion method [22],

$\varepsilon=\frac{H_{G}-H_{L}}{H_{G}}$

where $H_{L}$ and $H_{G}$ represented the height of the static liquid level and the bubbling liquid level in the PBR, respectively.

\subsubsection{Volumetric mass transfer coefficient}


The volumetric mass transfer coefficient is also a common parameter used to evaluate the performance of a PBR. It is interrelated with many factors, such as the superficial gas velocity, liquid viscosity, and bubble size. The volumetric mass transfer coefficient $\left(K_{L} a\right)$ is the product of the liquid phase mass transfer coefficient $\left(K_{L}\right)$ and the effective interfacial area $(a)$, which is difficult to measure separately in practical applications. In this study, the total mass transfer coefficient was measured using the dynamic method [23]. The equation was as follows: $\frac{d C_{\mathrm{L}}}{d t}=K_{L} a \boldsymbol{C}^{*}-C_{L}$

where $C^{*}$ was the dissolved oxygen (DO) concentration in saturated liquid phase and $C_{L}$ was the DO concentration at the time $t$ in the liquid phase. To quantify the effect of the bubble size on the mass transfer process, a mass transfer model that combined Higbie's penetration theory and a velocity-slip model was used to describe the mass transfer process of different bubbles in the PBR. The expression[24] was $K_{L}=2 \sqrt{D_{L} v_{s} / \pi d_{s}}$

where $K_{L}$ was the liquid mass transfer coefficient in $\mathrm{m} / \mathrm{s}, D_{L}$ was the axial dispersion coefficient of the liquid phase in $\mathrm{m}^{2} / \mathrm{s}$, and $v_{s}$ was the bubble slip velocity in $\mathrm{m} / \mathrm{s}$. The liquid axial dispersion coefficient and axial liquid velocity can be obtained from the empirical formula presented by R.

Krishna et al. [25]:

$$
D_{L}=0.31 V_{L}(0) D_{T}
$$

$V_{L}(0)=0.21\left(g D_{T}\right)^{1 / 2}\left(\frac{U_{\mathrm{g}}{ }^{3}}{g v_{L}}\right)^{1 / 8}$

In general, the expression for the gas-liquid effective interfacial area $a$ was 


$$
a=\frac{6 \varepsilon_{g}}{d_{\mathrm{s}}}
$$

\subsubsection{Three-phase mixed CFD model}

The mixing process of gas-liquid-algae is an important factor that affects the performance of a PBR. Efficient mixing ensures that a highly concentrated biomass to maintains a uniform distribution in its suspension, eliminates temperature unevenness, produces uniform distribution of the nutrient solution, and improves gas exchange, while it reduces the overlap of algae cells and the possibility of light suppression. According to the literature[26-29], CFD (computational fluid dynamics) simulations have been widely used to study the global mixing and flow regime in PBRs. Based on the Euler-Euler two-fluid model, numerical simulations of three-phase bubbling flow in the PBR were performed for the conditions of various bubble sizes. In these simulations, the Chlorella density was set to $1200 \mathrm{~kg} / \mathrm{m}^{3}$, and the particle diameter was assumed to be $5 \mu \mathrm{m}$ as measured by microscopic examination.

The mass conservation equation was as follows:

$$
\frac{\partial}{\partial t}\left(\alpha_{k} \rho_{k}\right)+\nabla \cdot\left(\alpha_{k} \rho_{k} \vec{v}_{k}\right)=0
$$

where $\alpha_{k}, \rho_{k}$, and $\vec{v}_{k}$, represented the volume fraction, density, and velocity vector of the $k^{\text {th }}$ phase, respectively.

The momentum conservation equation was as follows:

$$
\frac{\partial}{\partial t}\left(\alpha_{k} \rho_{k} \vec{v}_{k}\right)+\nabla \cdot\left(\alpha_{k} \rho_{k} \vec{v}_{k} \vec{v}_{k}\right)=-\alpha_{k} \nabla p-\nabla \cdot \bar{\tau}_{k}+\alpha_{k} \rho_{k} \vec{g}+\vec{F}_{k}
$$

In the momentum equation, $\bar{\tau}_{k}$ represented the stress tensor. According to the Boussinesq assumption, if treating the flow as isotropic turbulence, the expression for the stress tensor was 
as follows:

$$
\bar{\tau}_{k}=\alpha_{k} \mu_{k}\left(\nabla \vec{v}_{k}+\nabla \vec{v}_{k}^{T}\right)+\alpha_{k}\left(\lambda_{k}-\frac{2}{3}\right) \nabla \cdot \vec{v}_{k} \bar{I}
$$

where $\mu_{k}$ and $\lambda_{k}$ were the sheer and bulk viscosities of the $k^{\text {th }}$ phase. $\bar{I}, \vec{g}, P, \vec{F}_{k}$ were the unit matrix, the gravitational acceleration, pressure and interphase forces, respectively. It was generally believed that the interphase forces include the drag force $\vec{F}_{d}$, virtual mass force $\vec{F}_{v m}$, and lift force $\vec{F}_{\text {lift }}$, which were described by the following equations:

$$
\begin{aligned}
& \vec{F}_{d}=\frac{3}{4} \alpha_{k 1} \alpha_{k 2} \frac{\rho_{k 1}}{d_{\mathrm{s}}} C_{D}\left|\vec{v}_{k 1}-\vec{v}_{k 2}\right| \boldsymbol{\sigma}_{k 1}-\vec{v}_{k 2}- \\
& \vec{F}_{v m}=C_{v} \rho_{k 1} \alpha_{k 2}\left(\frac{d \vec{v}_{k 1}}{d t}-\frac{d \vec{v}_{k 2}}{d t}\right) \\
& \vec{F}_{\text {lift }}=C_{l} \rho_{k 1} \alpha_{k 2} \vec{\sigma}_{k 1}-\vec{v}_{k 2} \supseteq \nabla \times \vec{v}_{k 1}-
\end{aligned}
$$

where $k_{1}$ and $k_{2}$ represented the primary phase and the secondary phase .The term $\frac{d}{d t}$ denoted the phase material time derivative of the form. The default values were used for the interphase force model parameters. $d_{s}$ was the diameter of the bubbles. Based on the experimental observations, bubbles were assumed all in spherical shape, and aggregation and fragmentation among the bubbles were assumed to be negligible.

The standard $\kappa-\varepsilon$ model was used to calculate the mixture multiphase turbulence in the PBRs, assuming the flow was fully developed and the influence of intermolecular viscosity could be neglected. In this model, $\kappa$ equation was the turbulent kinetic energy equation and the $\varepsilon$ equation was the turbulent dissipation equation, which were solved by the following equations:

$$
\begin{aligned}
& \frac{\partial}{\partial t}\left(\kappa \rho_{k}\right)+\nabla \cdot\left(\kappa \rho_{k} U_{k}\right)=\nabla \cdot\left[\left(\mu+\frac{\mu_{t}}{\sigma_{\kappa}}\right) \nabla \kappa\right]+G_{\kappa}+G_{b}-\varepsilon \rho_{k}-Y_{M}+S_{\kappa} \\
& \frac{\partial}{\partial t}\left(\varepsilon \rho_{k}\right)+\nabla \cdot\left(\varepsilon \rho_{k} U_{k}\right)=\nabla \cdot\left[\left(\mu+\frac{\mu_{t, k}}{\sigma_{\varepsilon}}\right) \nabla \varepsilon\right]+C_{1 \varepsilon} \frac{\varepsilon}{\kappa}\left(G_{\kappa}+C_{3 \varepsilon} G_{b}\right)-C_{2 \varepsilon} \rho_{k} \frac{\varepsilon^{2}}{\kappa}+S_{\varepsilon}
\end{aligned}
$$


where $G_{\kappa}$ and $G_{b}$ represented the turbulent kinetic energy generated by the average velocity gradient and buoyancy effect, respectively. $Y_{M}$ represented the contribution of the pluses generated by compressible turbulence to the overall dissipation rate. $C_{1 \varepsilon}, C_{2 \varepsilon}, C_{3 \varepsilon}, C_{\mu}$ represented the turbulence model coefficients. $S_{\kappa}$ and $S_{\varepsilon}$ were customized source terms and the liquid-phase turbulent viscosity $\mu_{t}$ was expressed by the following equation: $\mu_{t}=\frac{C_{\mu} \rho_{k} \kappa^{2}}{\varepsilon}$

The main object of the simulation was to study the bubbling flow without internal components, so the two-dimensional unsteady-state simulations were performed using the solver in the ANSYS Fluent 12.0 commercial software package. The pressure-velocity coupling was characterized using the phase-coupled simple method. The discrete method for the volume fraction equation employed the quick format. The equation of momentum and the $\kappa-\varepsilon$ model were both discretisized with the second-order upwind scheme. The wall effect was based on the standard wall functions method. The inlet and outlet boundary conditions were respectively assumed to be the velocity-inlet and the pressure-outlet ( $1 \mathrm{~atm})$. In addition, the wall was defined as having the no-slip condition. The default value was used for every variable relaxation factor. The initial time step was $0.001 \mathrm{~s}$, and then increased to $0.01 \mathrm{~s}$ after $10 \mathrm{~s}$. When the residual of the variable was less than 0.001 , the calculation results were assumed to be sufficiently converged,as thus, the flow tended to achieve a periodic quasi-steady state.

\section{Results and discussion}

\subsection{Bubble sizes and distribution}



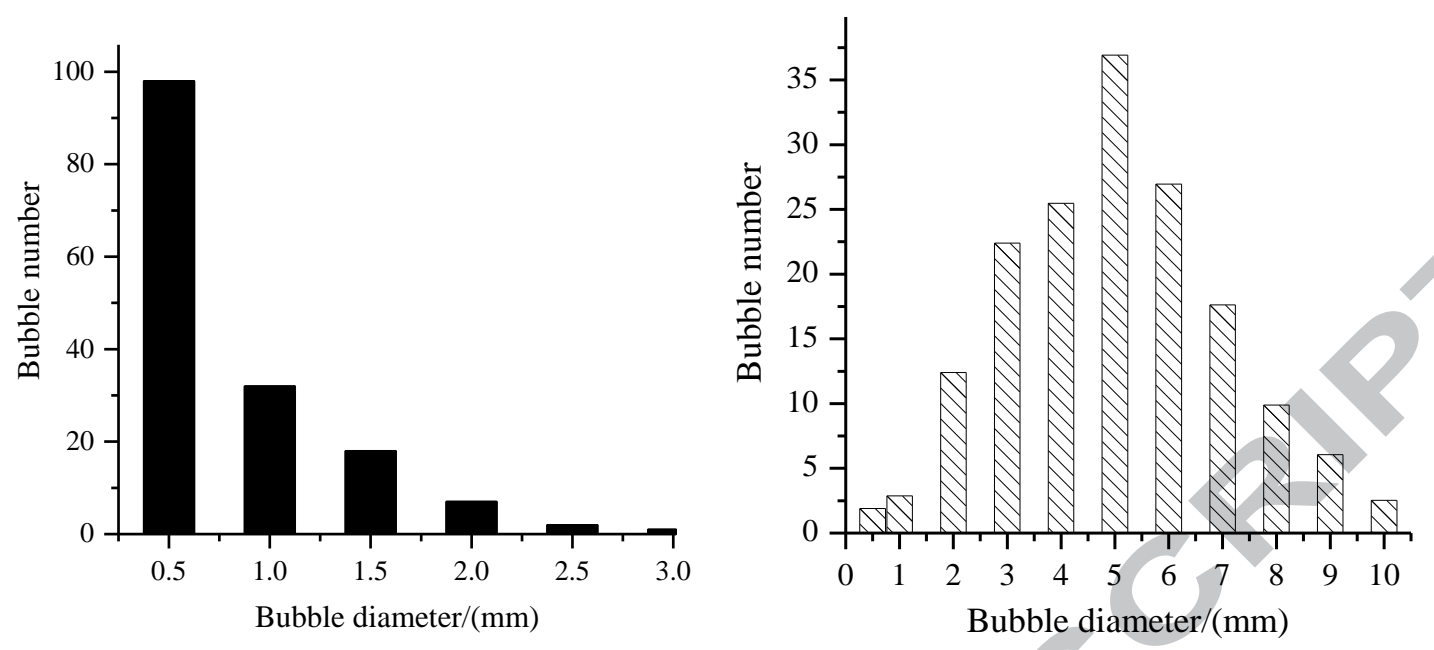

Fig. 1. Bubble size distribution of $\mathrm{S} 1$ (solid black columns) and $\mathrm{S} 2$ (striped columns) $\left(Q_{g}=10\right.$ $\mathrm{L} / \mathrm{min})$.

The time-averaged bubble size distribution of the $\mathrm{S} 1$ and $\mathrm{S} 2$ distributors at $10 \mathrm{~L} / \mathrm{min}$ were measured after processing the high-speed images using MATLAB, as shown in Fig. 1.

Furthermore, the effects of the different gas velocities on the time averaged bubble size distribution of the S1 and S2 distributors were studied. Fig. 2 showed that the bubbles produced by both $\mathrm{S} 1$ and $\mathrm{S} 2$ distributor increased in size as the intake air flow rate increased. In addition, the results revealed that most of the bubbles generated in the $\mathrm{S} 1$ distributor remained between 400 to $550 \mu \mathrm{m}$ at $2.5-10 \mathrm{~L} / \mathrm{min}$, i.e. the Sauter mean diameter of the bubbles was $523 \mu \mathrm{m}$ at $10 \mathrm{~L} / \mathrm{min}$. In contrast, the typical bubble size for the $\mathrm{S} 2$ distributor was $3.5-5.5 \mathrm{~mm}$ at the same gas flow rates, and the average diameter of the bubbles tended to stabilize at a Sauter radius of $5.2 \mathrm{~mm}$ at $10 \mathrm{~L} / \mathrm{min}$, as shown in Fig.2. 

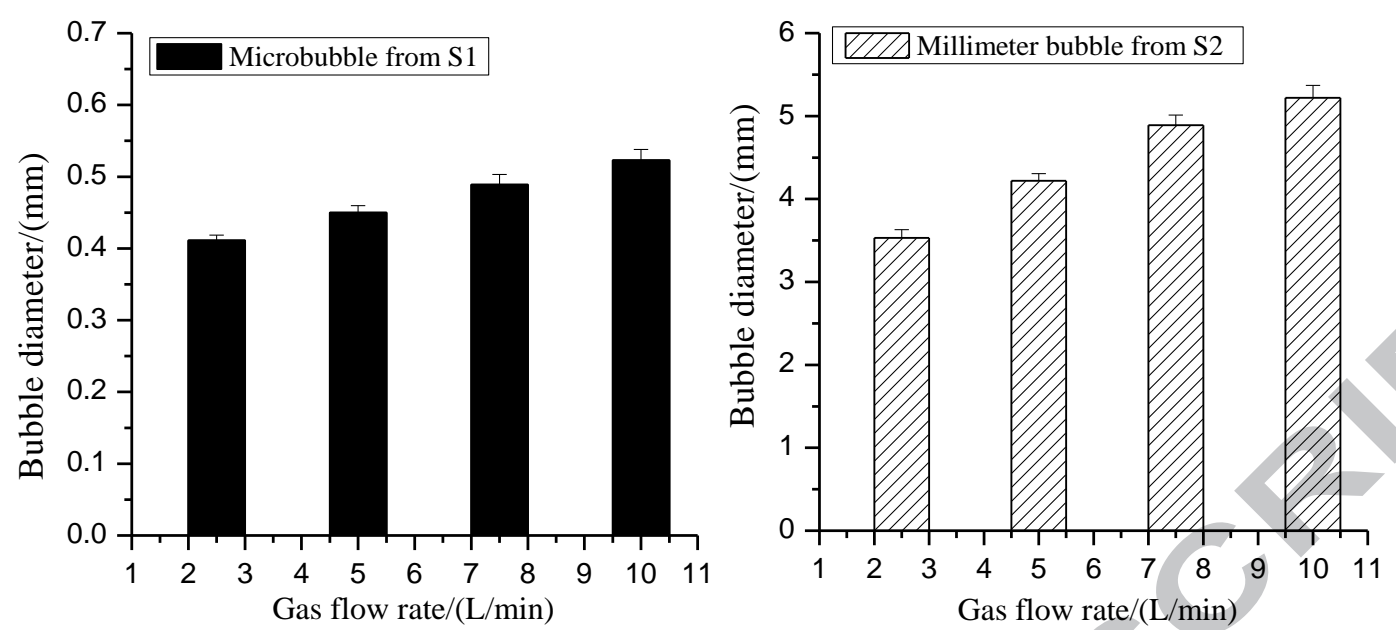

Fig. 2. Changes in the size of microbubbles (solid black columns) and millimeter bubbles (striped columns) with the increasing gas flow rate.

\subsection{Gas holdup}

The gas holdup was mainly affected by the intake air flow rate. As shown in Fig. 3, the gas holdup increased linearly with the gas flow rate for the range of $0-10 \mathrm{~L} / \mathrm{min}$, and the gas holdup in the microbubble bubbling state was greater than that in the traditional millimeter-bubble flow. Microbubbles rose much slower than millimeter bubbles, because the rising velocity depended on the buoyancy which was proportional to the bubble size. As a result, the smaller bubbles tended to stay longer and the gas holdup was greater when using S1 sparger at the same gas flow rate. 


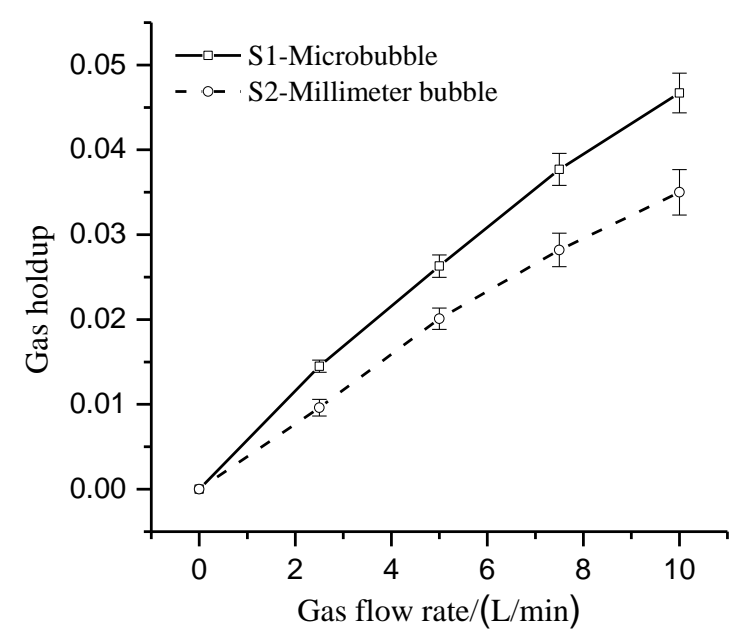

Fig. 3. Effects of microbubbles (solid line) and millimeter bubbles (dashed line) on the gas holdup as the gas flow rate increased.

\subsection{Mass transfer coefficient}

The effects of microbubbles and millimeter bubbles on the volumetric mass transfer coefficient were given by both the experimental measurements and the theoretical calculation model.The dynamic method was used to validate the mass transfer model, and one of the parameters, $v_{s}$ was predicted by the CFD simulations. Fig. 4 showed that the volumetric mass transfer coefficients of the liquid phase increased linearly as the gas flow rate increased. In particular, the mass transfer efficiency of microbubbles was much greater than that of millimeter bubbles because of their the smaller diameter. The advantages of microbubbles can be attributed to the following reasons: (a) Division of a single large bubble into several smaller, equally sized bubbles, which creates additional gas-liquid interfacial surface area. (b) The smaller bubbles have more time to carry out the mass transfer process. For the same gas flow rate, the total mass transfer rate is inversely proportional to the bubble size. Therefore, this mass transfer model was 
valid and beneficial to improving the performance of PBR.

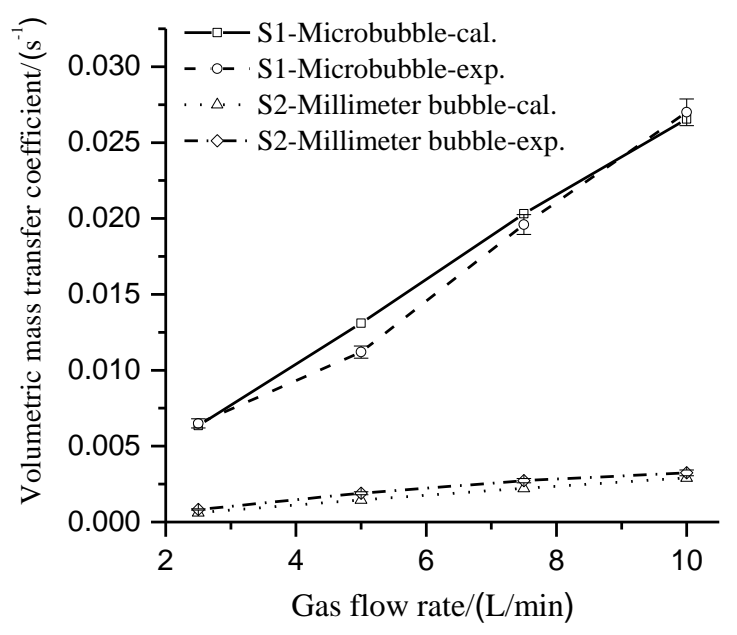

Fig. 4. Effects of the two types of bubbles on the volumetric mass transfer coefficient as the gas

flow rate increased.

\subsection{Effect of bubble size on illumination}

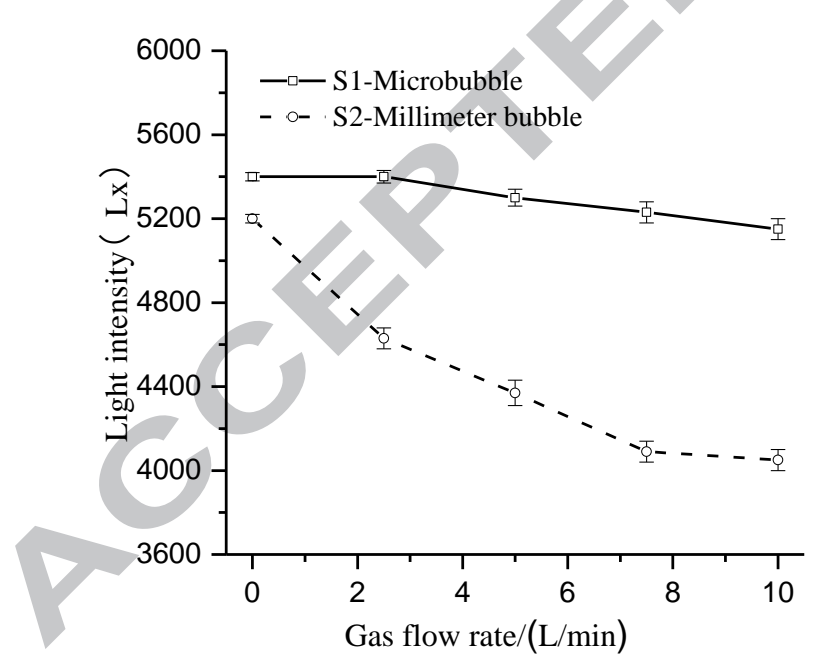

Fig. 5. Effects of microbubbles (solid line) and millimeter bubbles (dashed line) on the light intensity as the gas flow rate increased.

A light source is vital for photosynthesis in microalgae, and a synergistic effect exists 
between the flow field and the light field in PBRs. For instance, the bubble size could influence the light transmittance and attenuation. Here, we used the built-in light source to provide illumination, and the light intensity was measured at the outer wall of the plexiglas cylinder at a height of $20 \mathrm{~cm}$. The results in Fig.5 indicated that the attenuation of the light intensity increased with the gas flow rate. The light attenuation of the microbubbles was less than that of the millimeter bubbles, because the smaller bubbles scattered light more efficiently and the light field was more uniform. Employing microbubbles can both improve the light utilization efficiency and increase the gas-liquid interfacial area.

\subsection{Effect of bubble size on two types of microalgae cultivations}

Fig. 6 showed a comparison of the microscopic images of L3 Chlorella and CM7 cyanobacteria, and there was a significant difference between the sizes of the two algal cells. The strain of CM7 cyanobacteria investigated in this work was identified as the nostoc.sp strain PCC 7120 by the Applied Biosystems 3730xl DNA Analyzer, which exhibited in beadlike chains with 100-300 $\mu \mathrm{m}$ in length, whereas the cells of the L3 algae were spherical and approximately $5 \mu \mathrm{m}$ in length. Fig. 7 showed that the growth rate of the CM7 cyanobacteria was greater in the presence of millimeter bubbles. In contrast, the growth rate of the L3 Chlorella was greater when using microbubbles, mainly because the sizes of the L3 Chlorella cells were smaller than those of the microbubbles. Specifically, microbubbles produced a steady bubbling flow pattern, which was sufficient to provide a good mixing for $5 \mu \mathrm{m}$ particles. However, in the cultivation of the CM7 cyanobacteria, sedimentation and flow dead zones appeared within the PBR as the biomass increased, which indicated that the momentum of the microbubbles was too small to provide 
sufficient kinetic energy for mixing. Therefore, microbubbles were more beneficial for the applications in cultivating small-size algae.

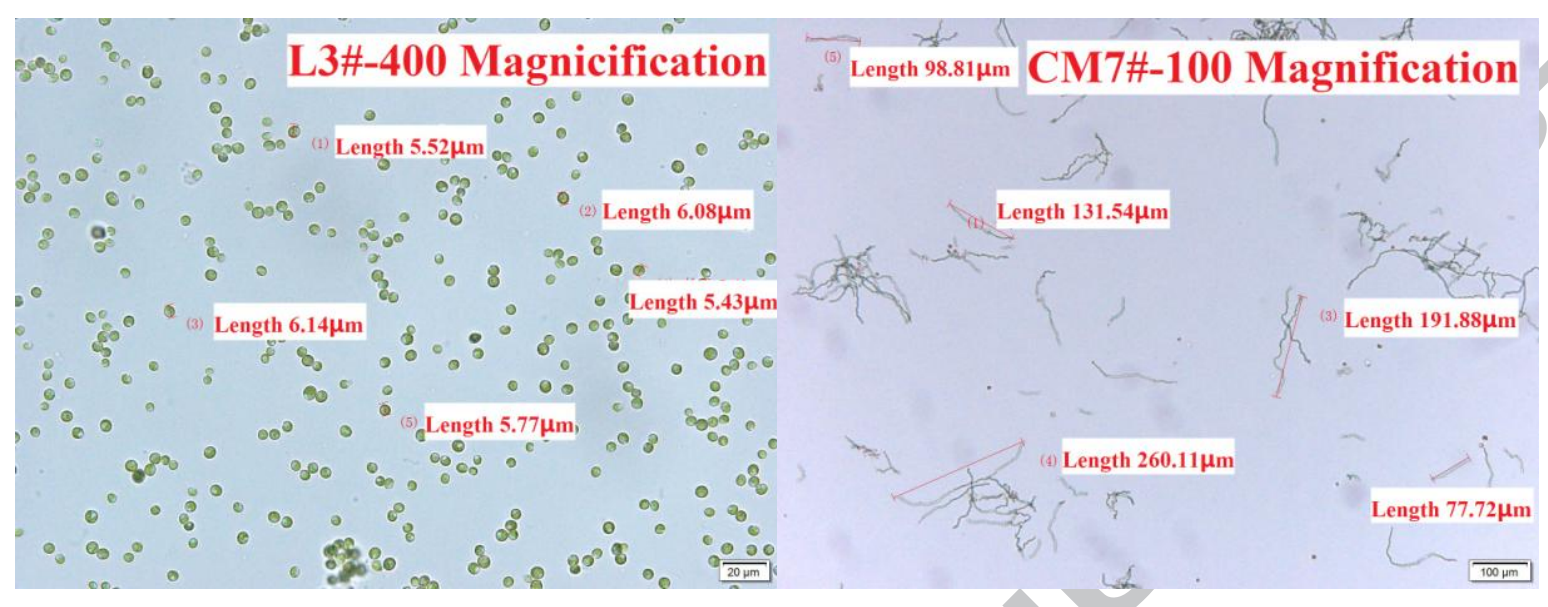

Fig. 6. Microscopic images of L3 Chlorella and CM7 cyanobacteria.

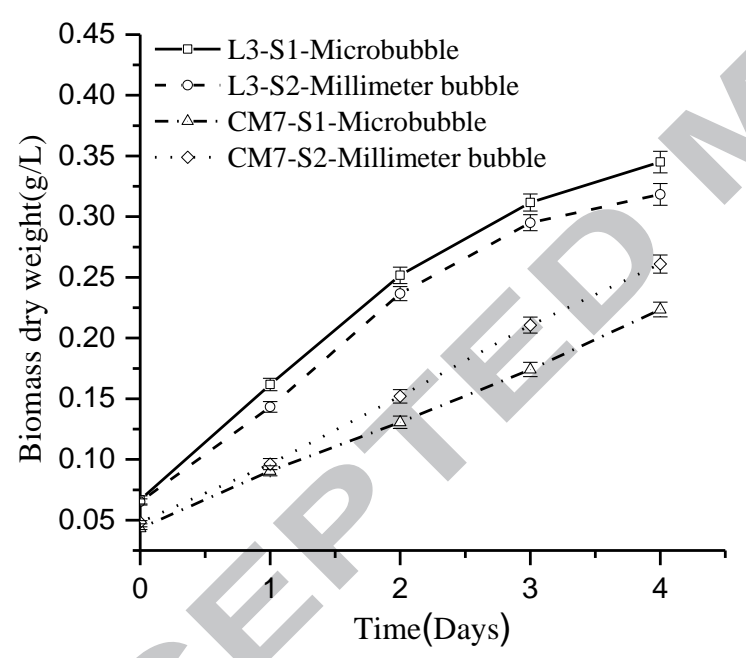

Fig. 7. Effects of bubble size on the growth curves of L3 Chlorella and CM7 cyanobacteria $\left(Q_{g}=5 \mathrm{~L} / \mathrm{min}\right)$

3.6 Effects of bubble size on the growth of Chlorella under different gas flow rates 

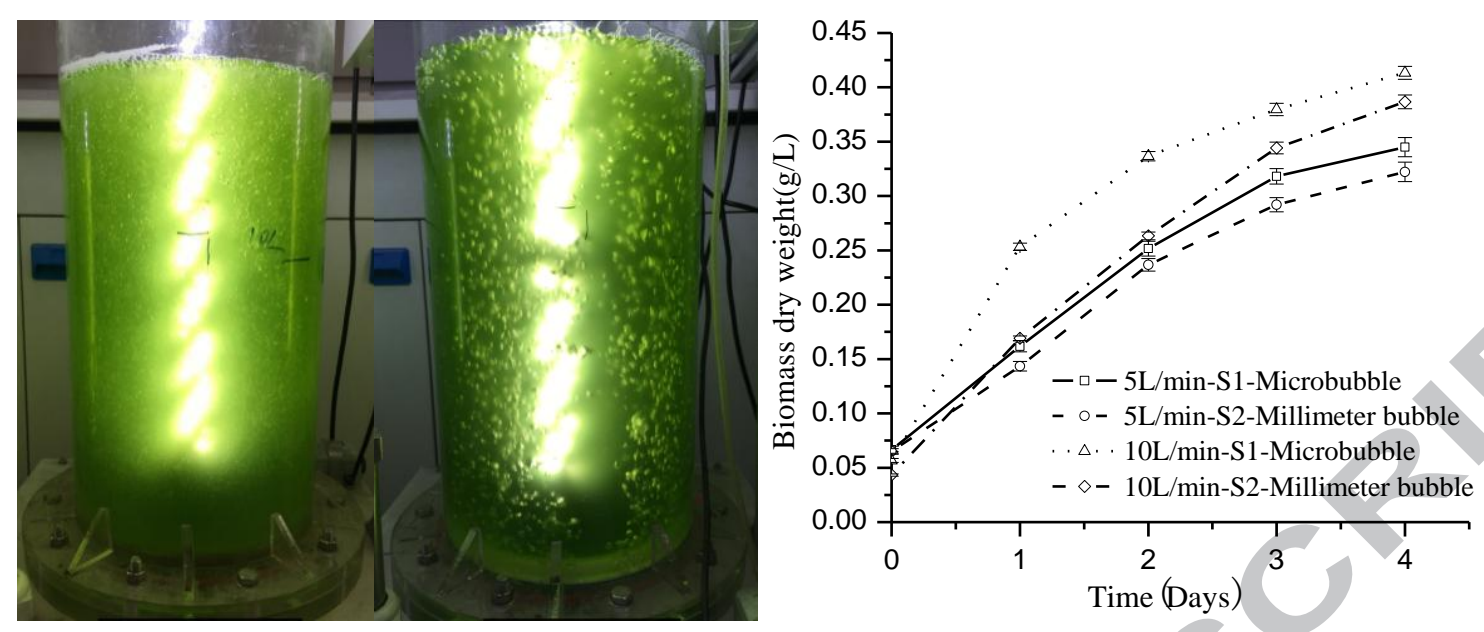

Fig. 8a (left). Chlorella cultures with different bubble sizes $\left(Q_{g}=10 \mathrm{~L} / \mathrm{min}\right)$.

Fig. 8b (right). Effects of the gas flow rate and bubble size on the growth curve of Chlorella.

While cultivating Chlorella (as seen in Fig. 8a), the influences of the gas flow rate and bubble size were studied. Fig. 8b showed that the growth efficiency of Chlorella increased with the gas flow rate. For the two types of bubbles, microbubbles increased the culture efficiency of Chlorella more significantly than the millimeter bubbles did because of the larger gas-liquid interfacial area associated with the microbubbles, which was conducive to improving the mass transfer and photo bio-reaction. In addition, at the gas flow rate of $10 \mathrm{~L} / \mathrm{min}$, the culture using microbubbles exhibited a high growth rate during the early stage, and then experienced a decreased rate during the later stage.A similar trend was found in the culture using millimeter bubbles. The decrease in the growth rate in the presence of millimeter bubbles was less than that in the presence of microbubbles. The decreased microalgae growth was caused by factors such as nutrient depletion, decreased light penetration and sedimentation of Chlorella in the PBR as the culture time increased. It was obvious that the increased biomass restricted the efficient mixing 
of microbubbles and the sedimentation might be more dominant, which can be seen from Fig. 9. The light intensities at the different heights were measured for the low and high concentrations of algal biomass cultivated with microbubbles and millimeter bubbles. When microbubbles were used, the algae solution was uniform at a concentration of $0.05 \mathrm{~g} / \mathrm{L}(\mathrm{dw})$ and the light intensity decreased as the height decreased at the higher concentration of $0.35 \mathrm{~g} / \mathrm{L}(\mathrm{dw})$, which meant that sedimentation of Chlorella occurred. However, the light attenuation varied little at the different axial positions when using millimeter bubbles. Furthermore, Fig. $8 \mathrm{~b}$ showed that the slope of the millimeter-bubble curve at the $3^{\text {rd }}$ and $4^{\text {th }}$ days was larger than that of the microbubble curve. Due to the high kinetic energy carried by the larger-size bubbles, the restriction of the bulk biomass on the flow mixing was alleviated, and a relatively better flow mixing was maintained.

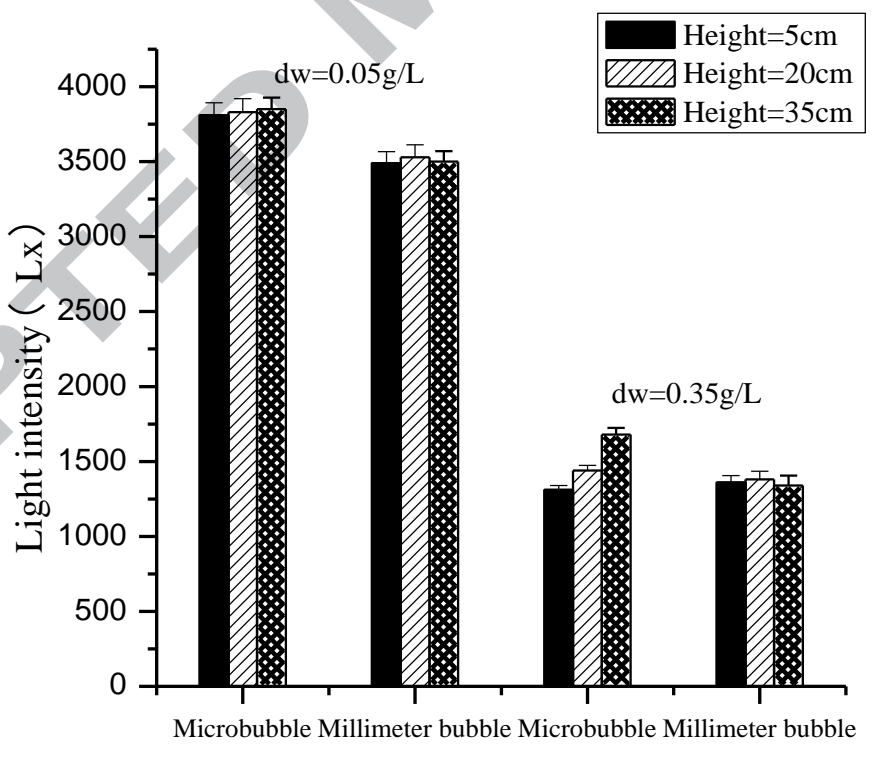

Fig. 9. Light intensity at the different heights with microbubbles and millimeter bubbles $\left(Q_{g}=10\right.$ $\mathrm{L} / \mathrm{min})$. 


\subsubsection{Effect of multiscale bubbles on the multiphase flow}
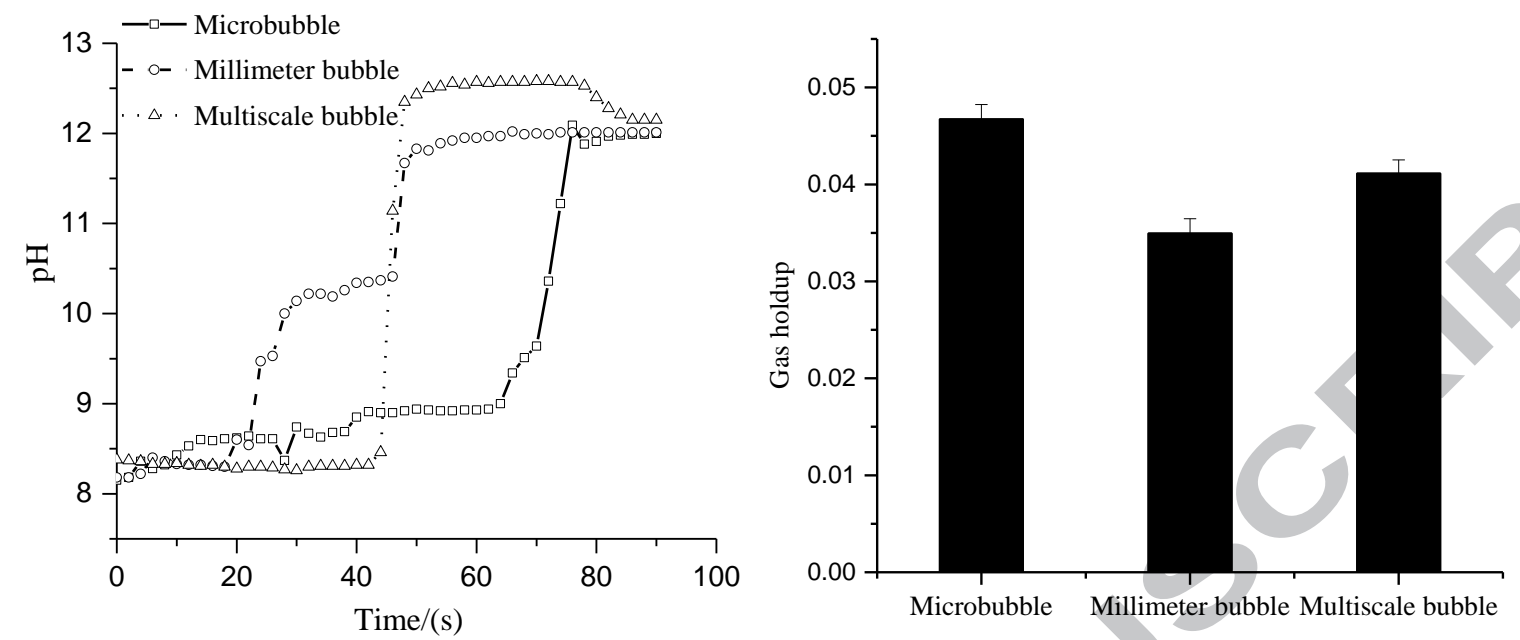

Fig. 10a (left). Effects of different types of bubbles on the residence time $\left(Q_{g}=10 \mathrm{~L} / \mathrm{min}\right)$.

Fig. 10b (right). Effects of different types of bubbles on the gas holdup $\left(Q_{g}=10 \mathrm{~L} / \mathrm{min}\right)$.

To study the characteristics of multiphase bubbly flow, $50 \mathrm{~mL}$ of sodium hypochlorite were injected into the bottom of the reactor as a tracer, and the $\mathrm{pH}$ of the liquid was recorded for three types of bubbles, at a gas flow rate of $10 \mathrm{~L} / \mathrm{min}$, the results were given in Fig. 10a. As demonstrated in the graph, the mixing time of gas and liquid phases by microbubbles supplied was longer, which indicated that microbubbles had a longer residence time. In addition. Fig. 10b showed that the effects of single-scale and multiscale bubble intake (i.e.,combining $5 \mathrm{~L} / \mathrm{min}$ microbubbles with $5 \mathrm{~L} / \mathrm{min}$ millimeter bubbles) on gas holdup, the light attenuation and the mass transfer coefficient were analyzed for a gas flow rate of $10 \mathrm{~L} / \mathrm{min}$. The gas holdup was the highest with only the microbubble intake, second-highest with the combined intake of multiscale bubbles, and the lowest with the traditional millimeter-bubble intake only. This pattern occurred because the gas holdup was proportional to the gas residence time, which increased with the decrease of bubbles size Therefore, the gas volume fraction was higher when the proportion of microbubbles 
increased. The experimental results of light attenuation in Fig. 11a revealed that the combined multiscale bubble intake reduced the light transmission ability to a certain extent, but the decrease was insignificant. Regarding the mass transfer coefficient, as illustrated in Fig. 11b, the calculated and experimental results of microbubbles and millimeter bubbles were basically consistent with the literature ${ }^{[15]}$. In addition, although the mass transfer ability decreased when multiscale bubbles were employed compared with the microbubble-only case, it was still much greater than the millimeter bubble-only case.
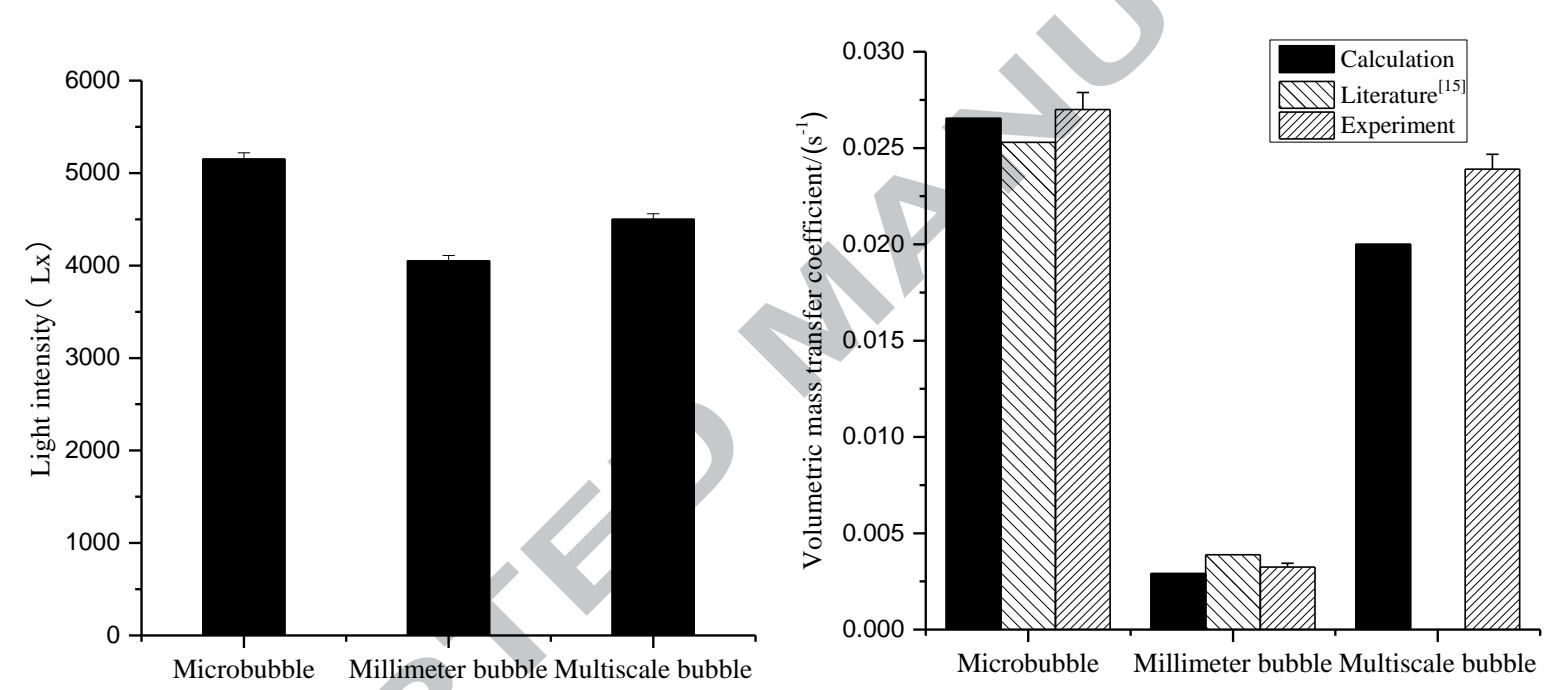

Fig. 11a (left). Effects of different types of bubbles on the light attenuation $\left(Q_{g}=10 \mathrm{~L} / \mathrm{min}\right)$.

Fig. 11b (right). Effects of different types of bubbles on the mass transfer coefficient $\left(Q_{g}=10\right.$ $\mathrm{L} / \mathrm{min})$.

\subsubsection{Effects of multiscale bubbles on the culture of Chlorella}



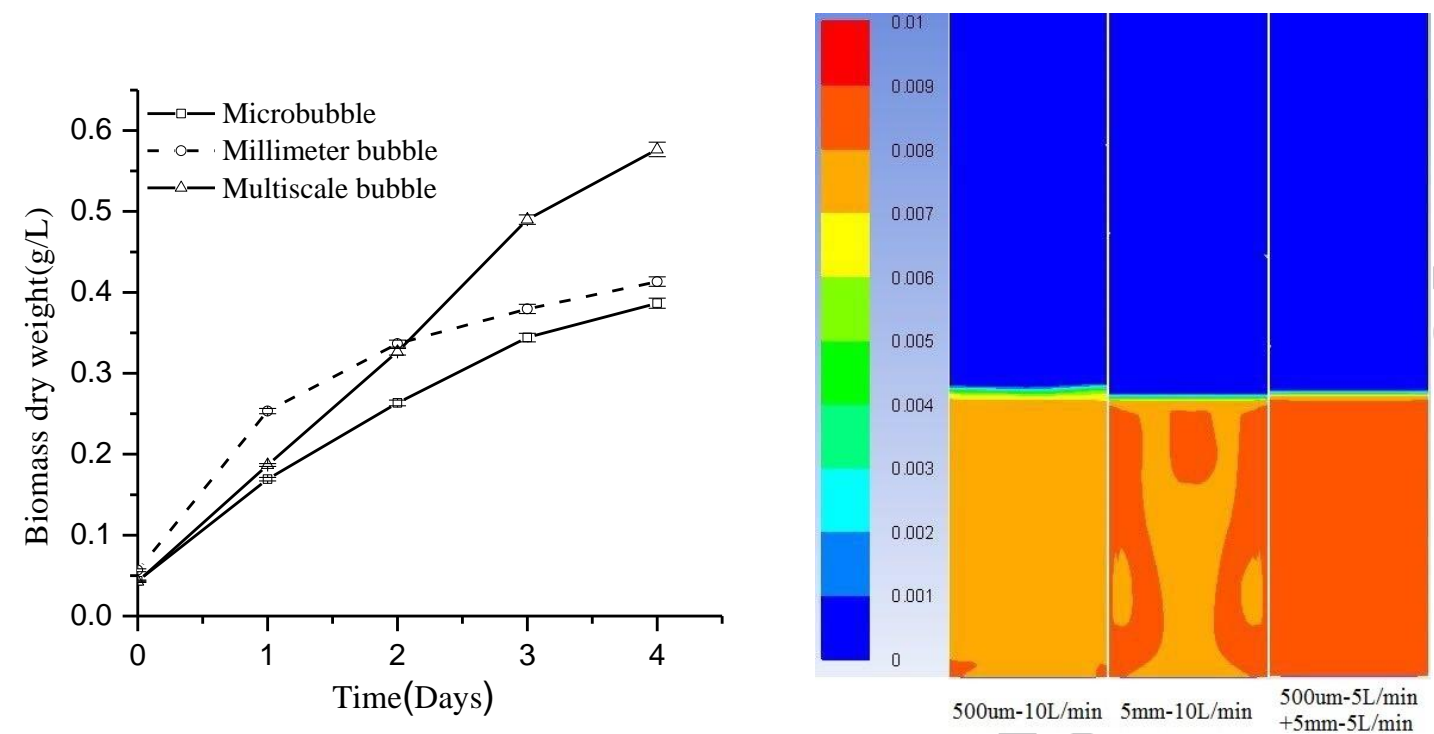

Fig. 12a (left). Effects of different types of bubbles on the growth curve of Chlorella by experiments $\left(Q_{g}=10 \mathrm{~L} / \mathrm{min}\right)$.

Fig. 12b (right). Effects of different types of bubbles on the gas-liquid-algae flow uniformity by CFD simulation $\left(Q_{g}=10 \mathrm{~L} / \mathrm{min}\right)$.

The time averaged distribution of Chlorella under bubbling conditions in the PBR at a dry weight of $0.5 \mathrm{~g} / \mathrm{L}$ (volume fraction $0.8 \%$ ) was investigated using CFD modeling. The results revealed that microbubbles could produce a uniform mixture, but certain degree of Chlorella deposition was observed at the bottom of the reactor. When millimeter bubbles were used, bubbles tended to gather in the center of the axial direction, led to uneven distribution of the algae solution, whereas the mixing of the algae solution was much more uniform when multiscale bubbles were used. Fig. 12a showed that the biomass growth rate with microbubbles was less than that with millimeter bubbles on the third day of culture. As mentioned above, the small size of microbubble resulted in a relatively weak transfer of momentum when interacting with a bulk biomass. Consequently, under the high concentration of microalgae, the mixing between the microbubbles 
and microalgae solution was constrained. For the combined gas intake of microbubbles and millimeter bubbles, the microbubbles enhanced the mass transfer and photosynthetic efficiency, meanwhile the millimeter bubbles helped to strengthen the flow mixing and eliminate the sedimentation of microalgae. Thus, the multiscale bubbles method was more robust for a uniform multiphase flow pattern as can be seen as Fig. 12b. Further, validation experiments for multiscale bubbles were performed, and the light intensities measured at the different positions were similar for the three different concentrations,as shown in Fig.13. The multiscale bubbles provided an uniform flow pattern for the biomass dry weight ranging from 0.05-0.5 g/L, and the experimental observations were agreed well with the CFD simulation results.Clearly, the biomass growth rate during the latter three days with the multiscale bubbles was greater than the rates obtained when using the single-scale bubbles, which proved that multiscale bubble was an optimal choice because it can significantly improve the photosynthetic growth efficiency of Chlorella.

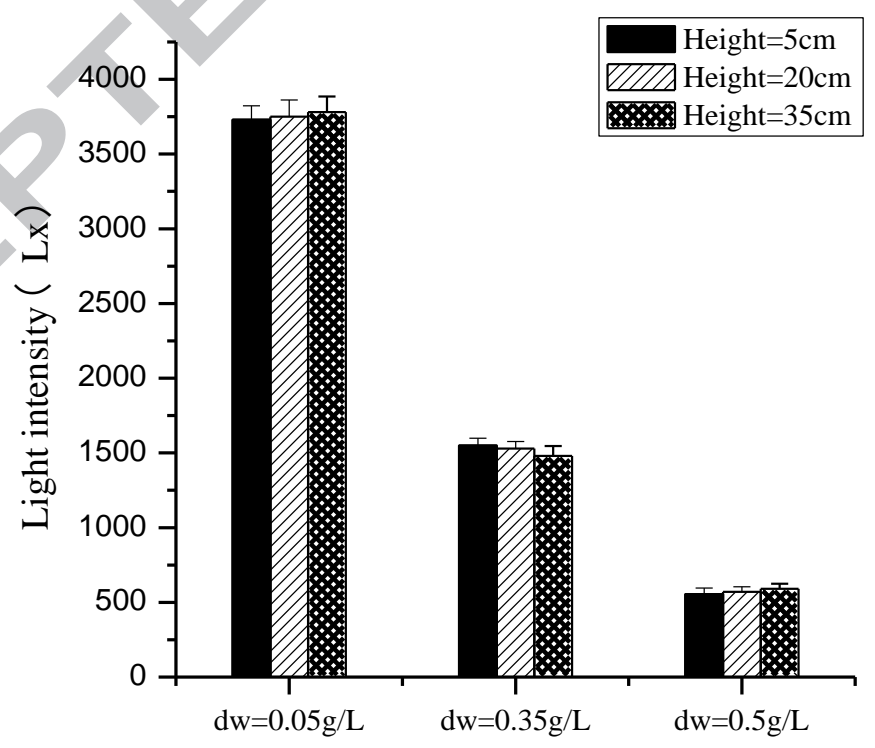

Fig. 13. Light intensity at the different heights with multiscale bubbles $\left(Q_{g}=10 \mathrm{~L} / \mathrm{min}\right)$. 


\section{Conclusions}

Based on the experimental observations and numerical simulations, the potential applications of three types of bubbles (microbubble, millimeter bubble, multiscale bubble) on the microalgae growth in a PBR were investigated. The following conclusions were drawn:

(1) Microbubbles can improve the gas holdup and mass transfer and strengthen the light utilization efficiency, thereby promoting the growth efficiency of microalgae. However, this effect applied successfully at the relative low biomass concentration.

(2) The choice of bubble size depended on the particle size of microalgae. The microalgae sedimentation phenomenon was prominent when microbubbles were employed for a large-particle-size cyanobacteria culture. For this culture, the algae growth efficiency was not as high as that when traditional millimeter bubbles were used. This result indicated that the uniform mixing and elimination of the biomass sedimentation were the primary factors for the optimal performance of gas bubbles. The utilization of microbubbles was more suitable for the application of small-particle-size algae such as Chlorella.

(3) For the cultivation of Chlorella, three types of bubbles were employed and their performances were analyzed. The growth efficiency of Chlorella initially increased and then decreased because of the weakened microbubble bubbling ability. In contrast, the larger-sized millimeter bubbles had higher momentum to lift the bulk biomass and to prevent sedimentation of microalgae. Therefore, to design more productive photobioreactors, the multiscale bubble method can be used in a PBR because of its dual-intensification function. The size distribution and proportion of multiscale bubbles should be adjusted based on the different species and the 
concentration of microalgae, which was a significant finding for industrial applications.

\section{Acknowledgement}

This work was carried out with the financial support from the SARI youth fund and the National Natural Science Foundation of China (No. 81400658). 


\section{References}

[1]L. Brennan, P. Owende, Biofuels from microalgae-A review of technologies for production, processing, and extractions of biofuels and co-products. Renewable and Sustainable Energy Reviews 14(2010), 557-577.

[2]S.J.Pirt, Y.K. Lee, M.R. Walach, M.W. Pirt, H.H.M. Balyuzi, M.J. Bazin, A tubular bioreactor for photosynthetic production of biomass from carbon dioxide: design and performance, Journal of Chemical Technology and Biotechnology 33(1983), 35-58.

[3]C.U. Ugwu, H. Aoyagi, H. Uchiyama, Photobioreactors for mass cultivation of algae, Bioresource Technology 99 (2008), 4021-4028.

[4]D.J. Farrelly, C.D. Everard, C.C. Fagan, K.P. McDonnell, Carbon sequestration and the role of biological carbon mitigation: a review, Renewable and Sustainable Energy Reviews 21(2013), 712-727.

[5]A. Concas, G.A. Lutzu, M. Pisu, G. Cao, Experimental analysis and novel modeling of semi-batch photobioreactors operated with Chlorella vulgaris and fed with $100 \%$ (v/v) $\mathrm{CO}_{2}$, Chemical Engineering Journal 213 (2012), 203 - 213.

[6]A. Concas, M. Pisu, G. Cao, Mathematical modelling of Chlorella Vulgaris growth in semi-batch photobioreactors fed with pure $\mathrm{CO}_{2}$, Chemical Engineering Transactions,32(2013),1021-1026.

[7]A. Concas, M. Pisu, G. Cao, Disruption of microalgal cells for lipid extraction through Fenton reaction: Modeling of experiments and remarks on its effect on lipids composition, Chemical Engineering Journal 263 (2015), 392 - 401. 
[8]A. Concas, M. Pisu, G. Cao, A novel mathematical model to simulate the size-structured growth of microalgae strains dividing by multiple fission, Chemical Engineering Journal 287 (2016), $252-268$.

[9]C. Posten, Design principles of photo-bioreactors for cultivation of microalgae, Engineering in Life Sciences 9(2009), 165-177.

[10]F.G.A. Fernández, J.M.F. Sevilla, J.A.S. Pérez, E.M. Grima, Y. Chisti, Airlift-driven external-loop tubular photobioreactors for outdoor production of microalgae: assessment of design and performance, Chemical Engineering Science 56(2001), 2721-2732.

[11]F.C. Rubio, F.G.A. Fernández, J.A.S. Pérez, F.G. Camacho, E.M. Grima, Prediction of dissolved oxygen and carbon dioxide concentration profiles in tubular photobioreactors for microalgal culture, Biotechnology and Bioengineering 62(1999), 71-86.

[12]Z.B. Zhang, H.Z. Tian, D.L. Wang, Y.R. Liu, J. He, M. Qi, F. Zhang, H.X. Luo, W.P. Ding, Z. Zhou, Intensification of interfacial mass transfer in gas-liquid reaction systems, Chemical Engineering 44(2016), 1-8.

[13]T.L. Zheng, Q.H. Wang, T. Zhang, Z.N. Shi, Y.L. Tian, S.S. Shi, N. Smale, J. Wang, Microbubble enhanced ozonation process for advanced treatment of wastewater produced in acrylic fiber manufacturing industry, Journal of Hazardous Materials, 287 (2015) 412-420.

[14]W.B. Zimmerman, M. Zandi, H.C. Bandulasena, V. Tesař, D. Gilmour, K. Ying, Design of an airlift loop bioreactor and pilot scales studies with fluidic oscillator induced microbubbles for growth of a microalgae Dunaliella salina, Applied Energy 88(2011), 3357-3369. 
[15]M.K. Al-Mashhadani, H.H. Bandulasena, W.B. Zimmerman, $\mathrm{CO}_{2}$ mass transfer induced through an airlift loop by a microbubble cloud generated by fluidic oscillation, Industrial \& Engineering Chemistry Research 51 (2012), 1864-1877.

[16]C. G. Khoo, M. K. Lam, K. T. Lee, Pilot-scale semi-continuous cultivation of microalgae Chlorella vulgaris in bubble column photobioreactor (BC-PBR): Hydrodynamics and gas-liquid mass transfer study,Algal Research 15 (2016), 65 - 76.

[17]O. N. Manjrekar, Y.J. Sun, L. He, Y. J. Tang, M. P. Dudukovic,Hydrodynamics and mass transfer coefficients in a bubble column photo-bioreactor, Chemical Engineering Science 168 (2017), $55-66$.

[18]H.X. YAN, Z.Z. HAN, H. ZHAO,et al., Characterization of calcium deposition induced by Synechocystis sp. PCC6803 in BG11 culture medium,CHIN. J. OCEANOL. LIMNOL., 32(2014),503-510.

[19]C.S. Sun, X.H.Zhang, H.W. Han, Image proceeding of micro-bubbles' size distribution in water, Opto-Electronic Engineering 39(2012), 7-11.

[20]R. Schäfer, C. Merten, G. Eigenberger, Bubble size distributions in a bubble column reactor under industrial conditions, Experimental Thermal and Fluid Science 26(2002), 595-604.

[21]A. Contreras, F. García, E. Molina, J.C. Merchuk, Interaction between $\mathrm{CO}_{2}$-mass transfer, light availability, and hydrodynamic stress in the growth of Phaeodactylum tricornutum in a concentric tube airlift photobioreactor, Biotechnology and Bioengineering 60(1998), $317-325$.

[22]Y. Chisti, Airlift Bioreactors. Elsevier, London, UK.(1989) . 
[23]F. Kargi, M. Moo-Young, Transport phenomena in bioprocesses, in: Moo-Young, M. (Ed.), Comprehensive Biotechnology, Pergamon Press, Oxford,UK(1985), pp. 21-22.

[24]A.A. Kulkarni, Mass transfer in bubble column reactors: effect of bubble size distribution, Industrial \& Engineering Chemistry Research 46 (2007), 2205-2211.

[25]R. Krishna, M.I. Urseanu, J.M. Van Baten, J. Ellenberger, Liquid phase dispersion in bubble columns operating in the churn-turbulent flow regime, Chemical Engineering Journal 78(2000), 43-51.

[26]J. Huang, F. Feng, M. Wan, J. Ying, Y. Li, X. Qu, R. Pan, G. Shen, W. Li, Improving performance of flat-plate photobioreactors by installation of novel internal mixers optimized with computational fluid dynamics, Bioresource Technology 182(2015), $151-159$.

[27]J. Pruvost, L. Pottier, J. Legrand, Numerical investigation of hydrodynamic and mixing conditions in a torus photobioreactor, Chemical Engineering Science 61(2006), 4476-4489.

[28]L.H.B. Zhao, et al.,CFD simulation of gas-liquid flow in bubble columns with different distributors, Modern Chemical Industry 32 (2012), 101-104.

[29]Q.Xiao, J. Wang,N.Yang,J.H. Li, Simulation of the multiphase flow in bubble columns with stability-constrained multi-fluid CFD models, Chemical Engineering Journal 329(2017),88-99. 


\section{Highlights:}

- Microbubbles can improve mass transfer and photo bio-reaction.

- The scale effect between bubbles and microalgae was studied.

- A multiscale bubble combination intake method was proposed.

- Multiscale bubbles can provide dual-intensification function.

- The multiscale bubbles method can enhance the growth efficiency of Chlorella. 